\title{
RESEARCH
}

Open Access

\section{Flow cytometry evaluation of CD14/CD16 monocyte subpopulations in systemic sclerosis patients: a cross sectional controlled study}

Laiana Schneider ${ }^{1,2^{*}}$ (D) Natália Aydos Marcondes ${ }^{3}$ (D) Vanessa Hax ${ }^{1}$, Isadora Flesch da Silva Moreira ${ }^{4}$, Carolina Yuka Ueda ${ }^{4}$, Rafaella Romeiro Piovesan ${ }^{4}$, Ricardo Xavier ${ }^{1,2}$ and Rafael Chakr ${ }^{1,2}$

\begin{abstract}
Background: Systemic sclerosis (SSC) is a chronic autoimmune disease characterized by vasculopathy and fibrosis, which can be subclassified into diffuse cutaneous (dSSC) and limited cutaneous (ISSC) subtypes. Previous studies suggest that an increase in monocytes can be a hallmark of various inflammatory diseases, including SSc. Our aim was to evaluate circulating blood monocyte subpopulations (classical, intermediate and non-classical) of SSc patients and their possible association with disease manifestations.

Methods: Fifty consecutive patients fulfilling the 2013 ACR/EULAR classification criteria for SSc were included in a cross-sectional study. Monocyte subpopulations were identified based on their expression of CD64, CD14 and CD16, evaluated by flow cytometry, and were correlated with the clinical characteristics of the patients; furthermore, the expression of HLA-DR, CD163, CD169 and CD206 in the monocytes was studied. Thirty-eight age- and sex-matched healthy individuals were recruited as a control group.

Results: SSc patients had an increased number of circulating peripheral blood monocytes with an activated phenotypic profile compared to healthy subjects. Absolute counts of CD16+ (intermediary and non-classical) monocyte subpopulations were higher in SSc patients. There was no association between monocyte subpopulations and the clinical manifestations evaluated.

Conclusion: We identified higher counts of all monocyte subpopulations in SSc patients compared to the control group. There was no association between monocyte subpopulations and major fibrotic manifestations. CD169 was shown to be more representative in dSSc, being a promising marker for differentiating disease subtypes.
\end{abstract}

Keywords: Systemic sclerosis, Monocytes, Monocyte subpopulations, Flow cytometry, Pathogenesis

\footnotetext{
* Correspondence: laia_schneider@hotmail.com

'Serviço de Reumatologia, Hospital de Clínicas de Porto Alegre, Universidade Federal do Rio Grande do Sul, 2350 Ramiro Barcelos St, Room 645, Porto

Alegre, RS 90035-903, Brazil

2Programa de Pós Graduação em Medicina: Ciências Médicas, Universidade

Federal do Rio Grande do Sul, Porto Alegre, Brazil

Full list of author information is available at the end of the article
}

(c) The Author(s). 2021 Open Access This article is licensed under a Creative Commons Attribution 4.0 International License, which permits use, sharing, adaptation, distribution and reproduction in any medium or format, as long as you give appropriate credit to the original author(s) and the source, provide a link to the Creative Commons licence, and indicate if changes were made. The images or other third party material in this article are included in the article's Creative Commons licence, unless indicated otherwise in a credit line to the material. If material is not included in the article's Creative Commons licence and your intended use is not permitted by statutory regulation or exceeds the permitted use, you will need to obtain permission directly from the copyright holder. To view a copy of this licence, visit http://creativecommons.org/licenses/by/4.0/. 


\section{Background}

Systemic sclerosis (SSc) is a chronic inflammatory systemic disease, mainly characterized by expansion of the secondary extracellular matrix due to an exaggerated protein production, especially collagen. Collagen is deposited as a result of the abnormal interaction between endothelial cells, mononuclear cells (lymphocytes and monocytes) and fibroblasts. Monocytes express several receptors, which monitor and detect environmental changes. Those cells present great plasticity and heterogeneity and have the ability to modify their functional phenotype in response to stimulation [1]. There seems to be a relationship between the monocytes/macrophages activation and migration [2, 3], and SSc pathogenesis [4].

Evidences from human and murine studies suggest that an increase in monocytes can be an indicator of various inflammatory diseases and that these cells could differentiate into inflammatory or anti-inflammatory subgroups [5]. For almost two decades studies have shown the presence of two subsets of cells: classical and non-classical monocytes. Classical monocytes, characterized by expression of CD14++CD16-, represent about $80 \%$ of all monocytes and have phagocytic function, with production of reactive oxygen species and secretion of pro- and anti-inflammatory cytokines in response to agonist stimulation of toll-like receptor-4 (TLR-4) [6]. Whereas non-classical monocytes, which express $\mathrm{CD} 16+$, have a higher activity in response to viruses and produce proinflammatory cytokines through TLR-8 and TLR-9 [7, 8].

In recent years, a third group of monocytes has been proposed, dividing the $\mathrm{CD} 16+$ population into nonclassical and intermediate monocytes (respectively, CD14+/-CD16++ and CD14+CD16+) [8-10]. These $\mathrm{CD} 16+$ monocytes appear to be related to alternative activated M2 macrophages, and their activation pathway showed that they are associated with $\mathrm{T}$ helper (Th) 2 response $[5,11]$. The differences between these monocyte subsets have been made clear, with distinct phenotypes and different functions, such as defense against pathogens, homeostasis and tissue repair $[9,10,12]$.

Evaluation of mannose receptor-1 (MRC1 or CD206) and CD163 (macrophage scavenger receptors) that are typical of M2 macrophage activation pathway, showed an association of these markers with tissue repair and fibrosis [13], and in SSc patients those markers are associated with high pulmonary artery pressure and increased mortality [14]. Other marker that may play a key role in the monocyte activation present in SSc is CD169 (sialic acid-binding Ig-like lectine I or Siglec-1), also known as sialoadhesin, which has been shown to have a higher expression in a subset of SSc patients monocytes [15]. Monocytes of SSc patients have a greater expression of these molecules, which is even higher in patients with tissue disease and pulmonary artery hypertension.
Peripheral blood monocytes heterogeneity still lacks a clear comprehension of its role in SSc pathophysiology and clinical manifestations. The aim of our study was to characterize the three monocytes subpopulations of SSc patients, analyze the expression of CD163, CD169, CD206 and HLA-DR (function and activation monocytes receptors), and their association with clinical findings.

\section{Methods \\ Patients}

Fifty consecutive patients fulfilling the 2013 ACR/ EULAR classification criteria for SSc [16] were recruited from the Outpatient Rheumatology Clinic of the Hospital de Clínicas de Porto Alegre, Brazil. Clinical form of disease was classified as diffuse SSc (dSSc) or limited SSc (ISSc) according to the revised classification of LeROY $[17,18]$. Medications under use were obtained from medical records and were confirmed during medical appointments. Patients receiving immunosuppressive drugs for SSc at the time of inclusion were carefully evaluated since those medications can influence monocytes counts and function [9]. Only patients that received cyclophosphamide treatment at least 6 months prior to the interview were included. Disease duration was defined as the time interval since the onset of Raynaud's phenomenon or skin symptoms (whichever came first). Severity of skin involvement was assessed using the modified Rodnan skin score [19]. Laboratory test results including serology, presence of autoantibodies and pulmonary function tests such as assessment of lung diffusion capacity for carbon monoxide (DLCO) and forced vital capacity (FVC) performed in the period of 3 months before or after the peripheral blood sample collection were obtained in medical records. Patients with scleroderma in overlap syndrome with other connective tissue disease and/or infectious diseases (HIV, hepatitis $\mathrm{C}$ or B) were excluded.

\section{Controls}

Thirty-eight subjects age- and sex-matched to the SSc patients, with no previous history of autoimmune or hematological diseases and a normal complete blood cell (CBC) count (hemoglobin $>11.0 \mathrm{~g} / \mathrm{dl}$; white blood cells $>$ $4 \times 10^{3} / \mathrm{mm}^{3}$ and $<12 \times 10^{3} / \mathrm{mm}^{3}$; and platelets $>150 \times$ $10^{3} / \mathrm{mm}^{3}$ ) were recruited from a local blood donation center and at a Primary Health Care Service unit. The criteria for inclusion were the same as those used in blood donation.

\section{Study design}

This was a monocentric cross-sectional study carried out from October, 2016, to May, 2017. 


\section{Flow cytometry protocol}

Peripheral blood samples were collected in ethylenediaminetetraacetic acid (EDTA) coated tubes. CBC was performed in a Sysmex XE-2100 ${ }^{\mathrm{mm}}$ (Sysmex, Kobe, JPN) hematological counter and the total amount of peripheral blood monocytes was determined.

Flow cytometry immunophenotyping (FCI) to identify and characterize monocyte subpopulations was performed less than $4 \mathrm{~h}$ after blood sampling. About 1,000, 000 cells were placed into polystyrene tubes and were subjected to surface staining by incubation with titrated amounts of fluorochrome-conjugated monoclonal antibodies (MoAbs) against CD14 APC-H7 (clone $\mathrm{M} \phi \mathrm{P} 9$ ), CD64 BV421 (clone 10.1), CD16 BV510 (clone 3G8), HLA-DR PerCP-Cy5.5 (clone G46-6), CD206 APC (clone 19.2), CD169 BB515 (clone 7-239) and CD163 $\mathrm{PE}$ (clone $\mathrm{GHI} / 61$ ) for $30 \mathrm{~min}$ at room temperature and protected from light. All MoAbs were purchased from BD Biosciences (San Diego, USA). Red blood cells were lysed by incubation with $2 \mathrm{ml}$ of lysing buffer (BD
Biosciences) for $10 \mathrm{~min}$. Samples were washed and resuspended in phosphate buffered saline (PBS). At least 30, 000 gated monocytes were acquired in an 8-color BD FACSCanto $^{\mathrm{TW}}$ II flow cytometer (BD Biosciences) using FACSDiva $^{\mathrm{m}}$ software (BD Biosciences). Flow cytometer technical parameters were automatically set with $\mathrm{BD}$ Cytometer Setup and Tracking Beads (BD Biosciences). Daily internal quality control (QC) was performed with IMMUNO-TROL Cells (BD Biosciences).

\section{Phenotypic analysis of monocyte subpopulations}

Figure 1 shows the gating strategy used. Initially, doublets were excluded and total nucleated cells were selected based on FSC (forward scatter) versus SSC (side scatter) distribution. Monocytes were gated based on cell size and complexity, as well as CD64+ expression [20], and were plotted in a CD14 versus CD16 graph in order to characterize all three monocyte subpopulations: classical monocytes (CD14++CD16-), intermediate monocytes $(\mathrm{CD} 14++\mathrm{CD} 16+)$ and non-classical monocytes

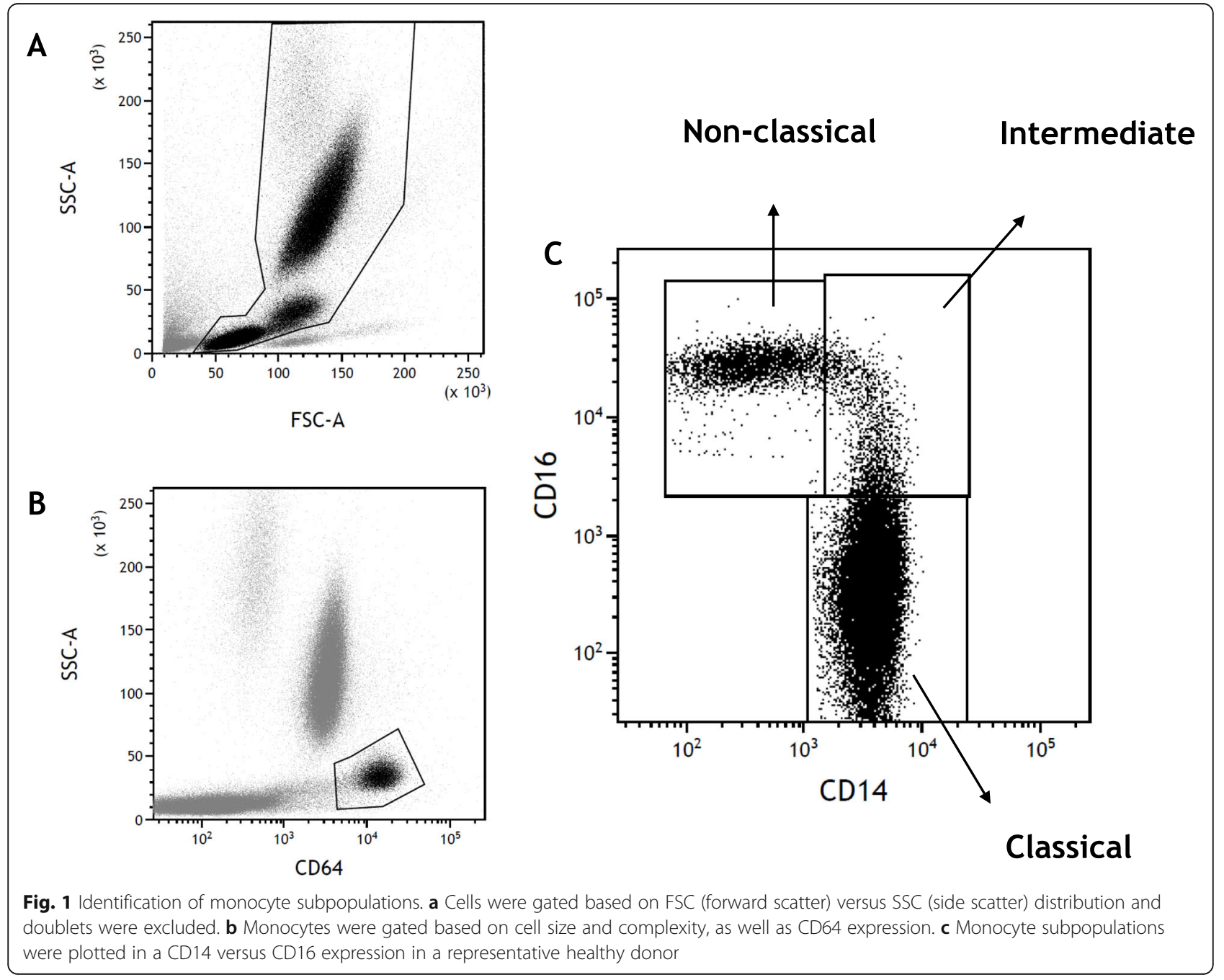


(CD14+/-CD16++). Fluorescence-minus-one (FMO) methodology was used to differentiate autoflourescent events from events with low concentrations of cell surface markers [21]. All values obtained in this analysis were considered FMO limit and all values of fluorescence above the limit were considered as true positive. Percentage of expression and mean fluorescence intensity (MFI) of any given marker within the defined monocyte subpopulation were recorded. All FCI data were analyzed using Kaluza Analysis Software (Beckman Coulter Life Sciences, Indianapolis, USA) by two experienced operators concomitantly.

\section{Compliance with ethical standards}

This study was approved by the Research Ethics Committee of the institution (CAAE number

Table 1 Demographic, clinical, and laboratory features of systemic sclerosis patients and controls

\begin{tabular}{|c|c|c|}
\hline & $\begin{array}{l}\text { Systemic sclerosis patients } \\
(n=50)\end{array}$ & $\begin{array}{l}\text { Healthy controls } \\
(n=38)\end{array}$ \\
\hline Female gender (\%) & $47(94.0)^{a}$ & $36(94.7)^{a}$ \\
\hline Age (years) & $57.2 \pm 12.8^{b}$ & $55.2 \pm 11.4^{b}$ \\
\hline European-derived (\%) & $40(80)$ & $24(68.6)$ \\
\hline \multicolumn{3}{|l|}{ Smoking status (\%) } \\
\hline Never & $25(50)$ & $23(65.7)$ \\
\hline Previous & $21(42)$ & $30(29.7)$ \\
\hline Current & $4(8)$ & $2(5.7)$ \\
\hline Disease duration (years) & $15(7-21)$ & \\
\hline Diffuse cutaneous involvement (\%) & $14(28.0)$ & \\
\hline Modified Rodnan skin score & $6(2.8-10.3)$ & \\
\hline Renal crisis & $4(8.0)$ & \\
\hline \multicolumn{3}{|l|}{ Gastrointestinal symptoms (\%) } \\
\hline Heartburn & $25(50.0)$ & \\
\hline Dysphagia & $26(52.0)$ & \\
\hline Diarrhea / constipation & $21(42.0)$ & \\
\hline DLCO (\% predicted) & $70.1 \pm 16.3$ & \\
\hline Reduced DLCO (<80\% predicted) & $52.9(15.9)$ & \\
\hline FVC (\% predicted) & $79.2 \pm 18.9$ & \\
\hline Reduced FVC (<80\% of predicted) & $22(44)$ & \\
\hline PASP & $31.7 \pm 14.4$ & \\
\hline Pulmonary hypertension (PASP $\geq 40 \mathrm{mmHg}$ ) (\%) & $5(10)$ & \\
\hline \multicolumn{3}{|l|}{ Autoantibodies (\%) } \\
\hline ANA & $39 / 50(78.0)$ & \\
\hline Anti-Ro/SSA & $1 / 45(2.2)$ & \\
\hline Anti-La/SSB & $0 / 45(0)$ & \\
\hline Anti-Scl-70 & $4 / 44(9.1)$ & \\
\hline RF & $35 / 50(75.0)$ & \\
\hline \multicolumn{3}{|l|}{ Medications (\%) } \\
\hline Prednisolone $<20 \mathrm{mg} /$ day & $6(12)$ & \\
\hline Mycophenolate mofetil & $4(8)$ & \\
\hline Previous methotrexate administration & $10(20)$ & \\
\hline Previous rituximab administration & $2(4)$ & \\
\hline Previous cyclophosphamide administration & $12(24)$ & \\
\hline
\end{tabular}

Data are shown as mean ( \pm standard deviation), median (interquartile ranges) or number $(\mathrm{n})$

ANA anti-nuclear antibodies, Anti-dsDNA anti-double-stranded DNA, Anti-Scl70 antitopoisomerasel, DLCO diffusion capacity of carbon monoxide, FVC forced vital capacity, PASP arterial systolic pressure on Doppler echocardiography, RF rheumatoid factor, SSC systemic sclerosis

${ }^{a}$ Mann-Whitney test, $p=1.000$

b Student's T test, $p=0.448$ 
49299515.0.0000.5327) and was conducted in accordance with the Declaration of Helsinki and current laws in Brazil. Participants were included after written informed consent.

\section{Statistical analysis}

Variables distribution was analyzed by Shapiro-Wilk's test and continuous variables were compared by Student's $\mathrm{T}$ or Mann-Whitney's U test, whereas proportions were compared by Pearson's chi-square or Fisher's exact test. ANOVA or Kruskall-Wallis, with a Tukey or Dunn correction test was used for multiple comparisons. Pearson or Spearman correlation test was used to evaluate association between variables. The statistical significance level adopted was $5 \%(p<0.05)$. Data analysis was carried out with SPSS version $21.0^{\circ}$ (IBM, Chicago, USA) and Prisma 8 software (Graphpad Inc. San Diego, USA).

\section{Results}

\section{Patients and controls characteristics}

Demographic data and clinical characteristics of SSc and healthy control $(\mathrm{HC})$ subjects are shown in Table 1. SSc patients mean age was 57.2 years and $94 \%$ were female. Limited form of disease was present in $72 \%$ of SSc patients. Median Rodnan skin score was $5(2.0-8.0)$ in ISSc patients and $11(3.8-16.8)$ in the dSSc patients $(p=$ 0.007). Pulmonary function also differed among disease subgroups when comparing forced vital capacity (FVC\%predicted) results: 1SSc subjects had higher mean values when compared to dSSc patients $(83.8 \pm 17.4 \%$ versus $67.5 \pm 18.2 \%, p=0.005$ ).

\section{Circulating monocyte subpopulations}

Main results of monocytes evaluation are show in Fig. 2. SSc patients had higher absolute and relative number of circulating monocytes when compared to HC (459.6 $(322.9-550.3) / \mathrm{mm}^{3}$ versus $273.9(179.3-352.2) / \mathrm{mm}^{3}$, $p<0.001)$, this difference remained significant when comparing ISSc or dSSc patients with HC independently (478.2 (342.9-555.5)/ $/ \mathrm{mm}^{3}$ versus $273.9(179.3-352.2)$ / $\mathrm{mm}^{3}, p<0.001$; and $387.4(310.4-551.1) / \mathrm{mm}^{3}$ versus $273.9(179.3-352.2) / \mathrm{mm}^{3}, p=0.002$, respectively) (data not shown). Among monocyte subpopulations, SSc patients had higher absolute counts of all subpopulations (classical, intermediate and non-classical) when compared to HC (classical monocytes 346.2 (260.9-450.8)/ $\mathrm{mm}^{3}$ versus $209.8(146.1-287.1) / \mathrm{mm}^{3}, p<0.001$; intermediate monocytes $38.2(24.6-47.1) / \mathrm{mm}^{3}$ versus 25.4 $(12.6-41.2) / \mathrm{mm}^{3}, \quad p=0.005$; non-classical monocytes $41.2(20.8-58.3) / \mathrm{mm}^{3}$ versus $28.3(11.5-46.1) / \mathrm{mm}^{3}, p=$ 0.006) (data not shown). Regarding disease subtype, ISSc patients presented increased absolute values of all monocyte subpopulations when compared to $\mathrm{HC}$ (classical monocytes $363.2 \quad(262.9-451.8) / \mathrm{mm}^{3}$ versus 209.8 (146.1-287.1) $/ \mathrm{mm}^{3}, p<0.001$ (A); intermediate monocytes $38.5(24.7-47.5) / \mathrm{mm}^{3}$ versus $25.4 \quad(12.6-41.2) /$ $\mathrm{mm}^{3}, p=0.009$ (B); non-classical monocytes 42.5 (20.8$69.0) / \mathrm{mm}^{3}$ versus $\left.28.3(11.5-46.1) / \mathrm{mm}^{3}, p=0.005\right)(\mathrm{C})$. While in dSSc patients, only classical monocytes had increased absolute counts compared to HC (209.8 (146.1$287.1) / \mathrm{mm}^{3}$ versus $\left.326.5(231.2-461.9) / \mathrm{mm}^{3}, p=0.003\right)$ (A). There was no difference in monocytes absolute or relative values between 1 SSc and dSSc subgroups $(p=$ 0.450 and $p=0.914$, respectively).

\section{Circulating CD16+ monocyte subpopulations}

When analyzing intermediate and non-classical monocytes as a single group of $\mathrm{CD} 16+$ monocytes, we identified a higher absolute count of this subpopulation in ISSc patients compared to HC $\left(79.9(53.4-103.5) / \mathrm{mm}^{3}\right.$ versus $\left.55.9(26.8-85.8) / \mathrm{mm}^{3}, p=0.003\right)$ (Fig. $2 \mathrm{~d}$ ).

\section{Monocyte subpopulations and clinical manifestations}

Absolute counts of monocyte subpopulations did not correlate with severity of cutaneous fibrosis assessed by the modified Rodnan skin score, pulmonary involvement assessed by DLCOc/VA, FVC and pulmonary artery systolic pressure, or disease duration when evaluated among the total of patients or in ISSc and dSSc subtypes (Fig. 3) (Additional files 1 and 2).
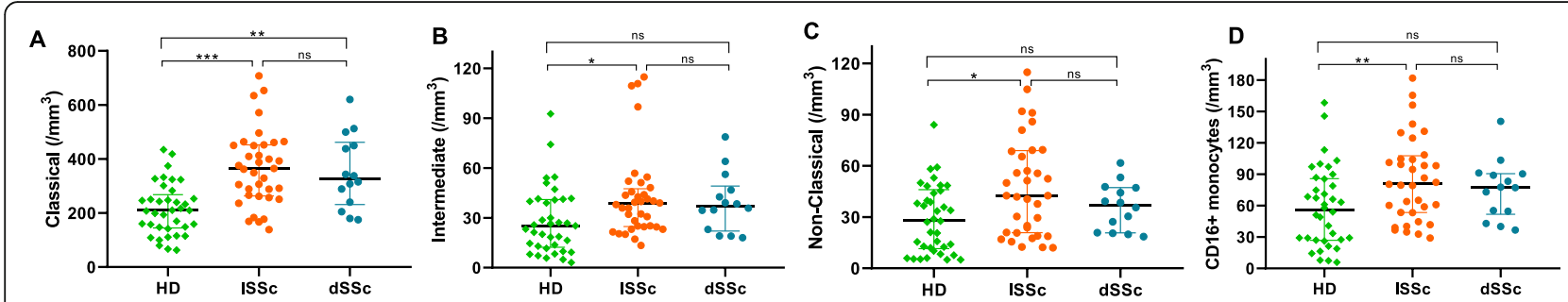

Fig. 2 Monocyte subpopulations according to their membrane expression of CD14 and CD16 in systemic sclerosis patients with limited (ISSC) $(n=36)$ and diffuse (dSSc) $(n=14)$ subtypes, and in healthy controls (HC) $(n=38)$. The absolute count of all monocyte subpopulations - classical $\mathbf{a}$, intermediate $\mathbf{b}$ and non-classical $\mathbf{c}$ - is increased in ISSC compared to HC. $\mathbf{d}$ When analyzing intermediate and non-classical monocytes as a single group of CD16+ monocytes, their absolute value is increased in ISSC compared to HC. ${ }^{*} p<0.05 ;{ }^{* *} p<0.01$; ${ }^{* * *} p<0.001$ 
Expression of surface receptors in monocyte subpopulations

Results of surface expression of HLA-DR, CD206, CD169 and CD163 receptors are shown in Fig. 4 and Additional file 3 . There were different expression patterns among the evaluated monocyte subpopulations. HLA-DR, an activation marker, was consistently expressed in all monocytes from SSc and $\mathrm{HC}$, and its intensity of expression was higher in all monocyte subsets from ISSc and dSSc patients when compared to control. CD206 expression varied among the monocyte subsets, the percentage of expression was higher in classical and intermediate monocytes of SSc patients when compared to $\mathrm{HC}$ and its MFI expression was lower in SSc as compared to HC. When analyzing SSc subgroups, percentage of CD206, the mannose receptor-1, expression was higher only in classical monocytes of ISSc patients compared to $\mathrm{HC}$ and in intermediate monocytes of ISSc and dSSc compared to HC; whereas MFI of CD206 expression was lower in both 1SSc and dSSc classical monocytes when compared to HC. CD163, macrophage scavenger receptor, was more expressed in classical (percentage and MFI) and intermediate (percentage) monocytes of SSc patients as compared to HC. In subgroup analysis, a difference in CD163 expression was demonstrated in classical monocytes of ISSc subjects compared to the HC group. CD169 receptor had a higher percentage of expression in SSc versus HC. When evaluating SSc subtypes, classical and intermediate monocytes from dSSc patients had higher MFI and percentage of CD169 expression than ISSc and $\mathrm{HC}$, whereas non-classical monocytes of dSSc patients had higher percentage of this marker expression (Fig. 4).

Further analyses were performed for comparison of the markers MFI of expression among the different monocyte subpopulations. Except for CD163 expression, the monocyte subpopulations exhibited distinct expression patterns when compared to one another,
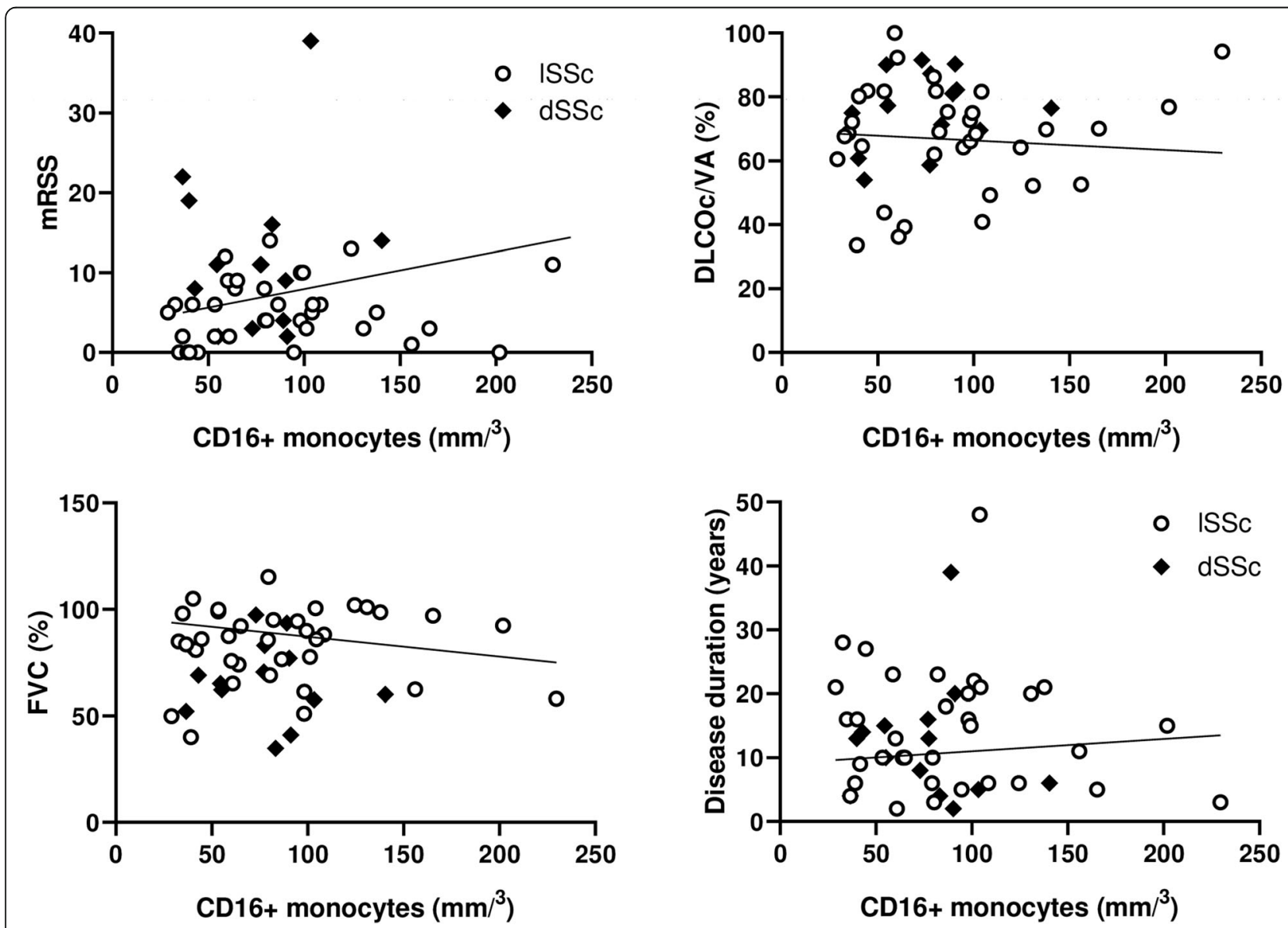

Fig. 3 Correlations regarding the CD16+ monocyte subpopulation. The CD16+ monocyte subpopulation of SSc patients ( $n=50$ ) and in the subgroup of patients presenting ISSC $(n=36)$ and dSSC $(n=14)$ was tested for association with cutaneous fibrosis assessed by the modified Rodnan skin score (mRSS), pulmonary involvement assessed by DLCOcNA and FVC. Disease duration was not associated with CD16 + values. DLCO: diffusion capacity for carbon monoxide; FVC: forced vital capacity 


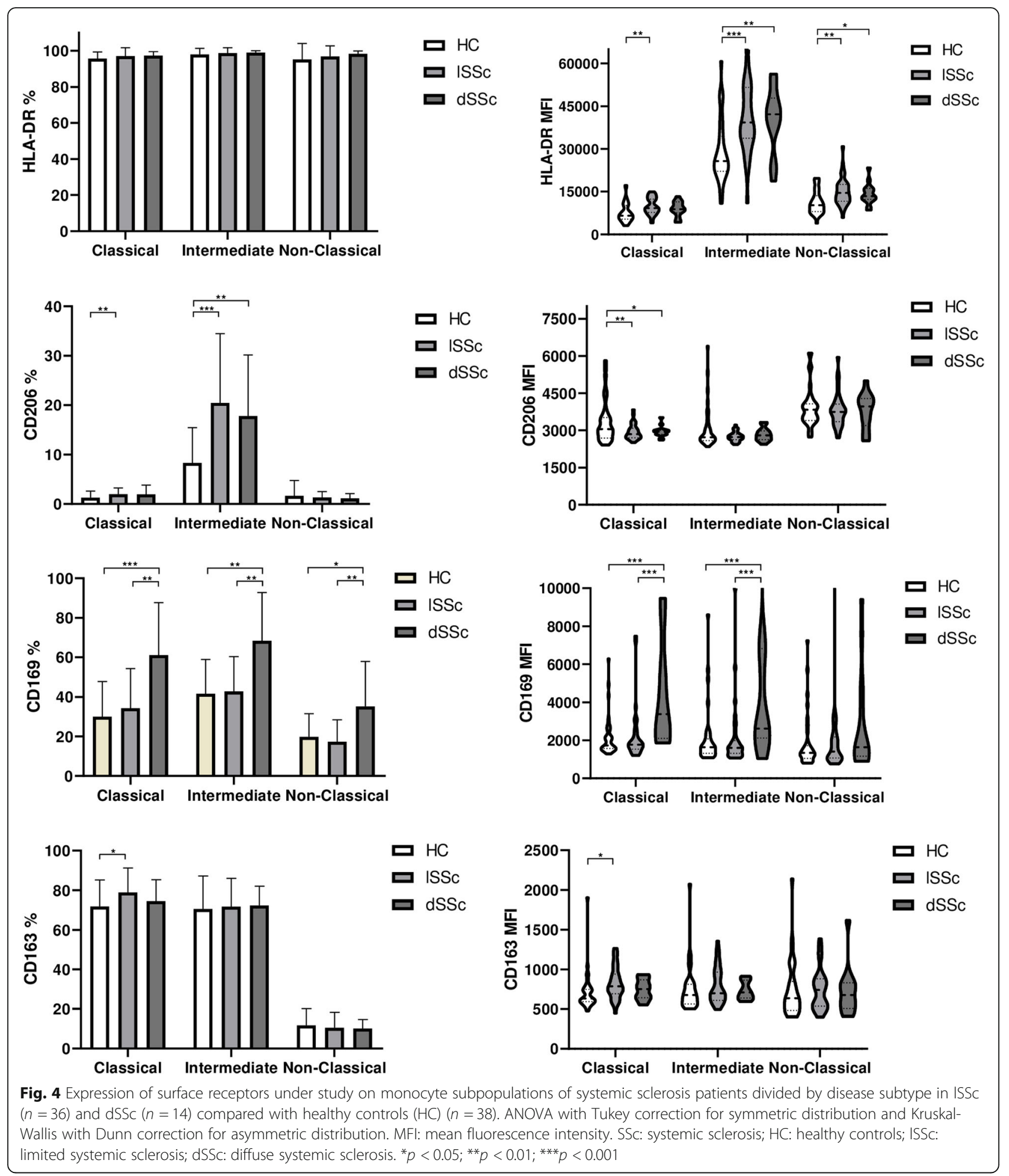


which was similar among patients and $\mathrm{HC}$ subgroups (Additional file 3).

\section{Discussion}

In our study, monocytes percentage and absolute count were significantly increased in SSc patients as compared to healthy subjects, regardless of disease subtype. Monocytosis in SSc patients has been reported previously [5, 22]. Assessment of CD14 and CD16 receptors expression allowed the identification of all three monocyte subpopulations, as already described for healthy individuals and some disease states [9, 23-25]. However, our study is the first to describe these subpopulations in SSc patients, using specific markers CD14 and CD16 for such distinction according to positivity and MFI of expression.

We identified an increase in all monocyte subpopulations in SSc patients, which was even more significant in patients with ISSc. Interestingly, ISSc patients showed an increase of all three monocyte subpopulations compared to controls, whereas in dSSc patients that increase was at expense of classical monocytes, indicating that in this form of disease the CD16+ monocyte subpopulations does not increase. Recent studies in SSc patients have shown an increase in CD16+ monocytes in the peripheral blood of these patients, however, these studies did not distinguish between intermediate and non-classical subpopulations $[5,22]$.

Previous studies have shown a significant correlation of the absolute CD16+ monocyte subpopulation count and severity of skin fibrosis and pulmonary fibrosis [5, 26]. In our study, we did not find correlations between monocyte subpopulations and the clinical manifestations evaluated. This difference may be related with intermediate and non-classical monocyte subpopulations grouped into CD16+ monocytes. Although differences among intermediate and non-classical monocyte subsets have been stablished $[9,10,12]$, there is a strong proximity between them [9], suggesting a direct developmental relationship, where gain of CD16 expression and decrease in CD14 expression may be indicative of maturation [27] and inflammatory activation for these cells to be recruited to the periphery, where they begin to act in localized inflammatory processes [5]. In addition, there are resident tissue macrophages originated from the yolk sac or from fetal liver progenitors [28], that have long tissue life and self-renewing ability by in situ proliferation, even in the absence of recruitment from circulating monocytes [29-31]. The role of these macrophages in tissues during inflammation is still unclear and further research is needed on the role of circulating monocytes for their activation and proliferation, especially in inflammation models.

Our data corroborate the results obtained by Lescoat et al. (2017) and Trombetta et al. (2018) concerning the increased amount of circulating monocytes with CD16+ monocytes as the main representative monocyte subset in SSc patients [5, 32]. Nevertheless, our results differ regarding correlations with clinical manifestations. The fact that we did not find a correlation of monocyte subsets with clinical manifestations reinforces the hypothesis of cellular modulation and activation in loco, since the different tissues are able to direct the inflammatory response in order to exacerbate inflammation with proinflammatory mediators or into resolution and repair [33]. Hence, findings of monocyte/macrophage activation in the bloodstream may not be observed at the tissue level, specifically, in internal organs affected by SSc.

HLA-DR is a cell activation marker, so it is consistent to find a greater expression intensity of this marker in patients with autoimmune diseases when compared to normal subjects. We found an increase in MFI expression of HLA-DR in all monocyte subpopulations of SSc patients compared to HC. MFI of HLA-DR expression in CD16+ monocyte subpopulations (intermediate and non-classical) was significantly higher than that of CD16- classical monocytes, indicating a greater activation of CD16+ antigen-presenting cells, which is expected in an autoimmune state.

Other molecules, such as capture receptor CD163 and CD206 were also associated with M2 macrophages and could be involved into further differentiation processes. A previous study comparing the expression of monocyte and macrophage markers in peripheral blood and skin biopsy specimens showed higher percentages of circulating cells expressing CD204, CD163 and CD14, furthermore these findings were correlated with skin involvement [22].

Additionally, circulating CD14+ monocytes/macrophages with CD206 coexpression have been shown to be associated with pulmonary artery hypertension [13]. It has also been shown that macrophages derived from circulating monocytes have coexpression of CD206, CD163 and CD169, and that the expression of these markers is significantly higher in patients with pulmonary interstitial disease [3]. In our study, a higher percentage of classical and intermediate monocytes with CD206 expression were identified in SSc patients compared to HC, which demonstrates a greater amount of M2-like cells in those monocyte subgroups of SSc patients.

Using a flow cytometry approach for assessing surface markers from circulating monocytes/macrophages, Soldano et al. (2018) demonstrated that SSc patients have higher expression of the M2 related markers CD14, CD206, CD163 and CD204; or that there is a coexpression of M1 and M2 surface markers in those cells [34]; providing evidence of plasticity in the expression of this markers in circulating monocyte/macrophage and that monocytes can evolve to exhibit features that are shared by more than one macrophage population. 
Previous studies have suggested that CD169 may be a phagocyte activity marker in inflammatory disorders. CD169 has been identified as a marker of highly pathogenic phagocytes in multiple sclerosis patients. Also, an increased expression of Siglec-1 on peripheral blood monocytes as well as its role in cell reactivity to autoantigen has been shown in rheumatoid arthritis [35, 36]. CD169 expression in renal macrophages has been correlated with proteinuria and tissue damage in glomerulonephritis [37], however its expression in peripheral blood cells was not assessed. CD169 expression has also been associated with involvement in the immune system regulation [38]. Biological functions of CD169 in macrophages and monocytes have yet to be elucidated. In our study, there was an increased expression of CD169 in classical monocytes from SSc patients compared to controls, and CD169 expression was higher in all monocyte subpopulations of dSSc patients compared to ISSc and controls. This finding could represent a greater disease activity in this subgroup of patients.

As to the use of medications, no association of these with subpopulations of monocytes was found. There were no differences between patients monocyte subpopulations regarding corticosteroids use $(n=6)$. Patients included in the study used low doses (less than $20 \mathrm{mg}$ per day), similar to previous reports [5].

Our study has some limitations. First of all, the patient population appears to be relatively small. However, there were many restrictions for patient inclusion in order to minimize treatment interference and disease overlap. Evaluation of exclusively circulating cells is also a limitation. There is a need for simultaneous assessment of circulating cells and tissue biopsies to better understand the participation of macrophages derived from circulating monocytes in tissue fibrosis. We have evidence that monocytes and macrophages actually participate in SSc pathogenesis and that there are slight differences between tissues [1, 39], nevertheless, we need better strategies to analyze these cells and studies assessing different tissues and peripheral blood to determine whether these macrophages are derived from circulating monocytes or other cells. In addition, our findings should be interpreted in light of our sample characteristics. Considering the pathophysiological and clinical changes observed in SSc over time, different monocyte subpopulations could be observed in early phases of the disease. Also, monocytes activation may be influenced by different levels of immunosuppression and distinct mechanisms of action of immunosuppressive drug treatment.

\section{Conclusions}

In conclusion, our results showed that SSc patients have increased absolute counts of all three monocyte subpopulations and that there is no difference among monocyte subpopulations between ISSc and dSSc disease subtypes. Monocytes from SSc patients presented a more activated phenotypic profile which was suggestive of M2 cells, with higher expression of CD206. Opposing to previous reports, we did not find an association between monocyte subpopulations and major fibrotic manifestations. The intermediate and non-classical monocyte subpopulations of SSc patients have increase expression of HLADR. CD169 appears to be more representative marker for dSSc subtype, however, more studies are necessary to confirm that this is a good marker for differentiation of disease subtype.

\section{Abbreviations}

CBC: Complete blood cell; DLCO: Lung diffusion capacity for carbon monoxide; dSSc: Diffuse cutaneous systemic sclerosis;

EDTA: Ethylenediaminetetraacetic acid; FCl: Flow cytometry immunophenotyping; FMO: Fluorescence-minus-one; FSC: Forward scatter; FVC: Forced vital capacity; HC: Healthy control; ISSc: Limited cutaneous systemic sclerosis; MFI: Mean fluorescence intensity; MoAbs: Monoclonal antibodies; MRC1: Mannose receptor-1; PBS: Phosphate buffered saline; QC: Quality control; Siglec-1: Sialic acid-binding Ig-like lectine I; SSc: Systemic sclerosis; SSC: Side scatter; Th: T helper; TLR: Toll-like receptor

\section{Supplementary Information}

The online version contains supplementary material available at https://doi. org/10.1186/s42358-021-00182-8.

Additional file 1: Table S1. Spearman correlation coefficient values between monocyte subpopulations and clinical data.

Additional file 2: Table S2. Analysis of the absolute of CD16 + in relation to the time of disease separated by tercil.

Additional file 3: Table S3. Comparison of the markers MFI of expression among the different monocyte subpopulations.

\section{Acknowledgements}

We would like to thank Fabiane Spagnol Pedrazzani, at the Department of Specialized Diagnostics of the Hospital de Clínicas de Porto Alegre, for her assistance in the design of the technical parameters for the flow cytometry protocol.

\section{Authors' contributions}

$L S, V H, R X$ and RC designed the project. LS, NAM, VH, IFSM, CYU and RRP collected the data and were responsible for data analysis. LS standardized and performed the flow cytometry experiments. LS and NAM wrote the manuscript. VH, RX and RC reviewed the manuscript. All authors read and approved the final manuscript.

\section{Funding}

We are grateful to the Fundo de Incentivo à Pesquisa e Eventos do Hospital de Clínicas de Porto Alegre (FIPE/HCPA) and the Conselho Nacional de Desenvolvimento Científico e Tecnológico (CNPq) for the financial support.

\section{Availability of data and materials}

The datasets used and/or analysed during the current study are available from the corresponding author on reasonable request.

\section{Declarations}

Ethics approval and consent to participate

This study was approved by the Research Ethics Committee of the institution (CAAE number 49299515.0.0000.5327) and was conducted in accordance with the Declaration of Helsinki and current laws in Brazil. Participants were included after written informed consent. 


\section{Consent for publication}

Not applicable.

\section{Competing interests}

The authors declare that they have no competing interests.

\section{Author details}

'Serviço de Reumatologia, Hospital de Clínicas de Porto Alegre, Universidade Federal do Rio Grande do Sul, 2350 Ramiro Barcelos St, Room 645, Porto Alegre, RS 90035-903, Brazil. ²Programa de Pós Graduação em Medicina: Ciências Médicas, Universidade Federal do Rio Grande do Sul, Porto Alegre, Brazil. ${ }^{3}$ Laboratório Zanol, Porto Alegre, Brazil. " Faculdade de Medicina, Universidade Federal do Rio Grande do Sul, Porto Alegre, Brazil.

\section{Received: 20 November 2020 Accepted: 27 April 2021} Published online: 22 May 2021

\section{References}

1. Taroni JN, Greene CS, Martyanov V, Wood TA, Christmann RB, Farber HW, et al. A novel multi-network approach reveals tissue-specific cellular modulators of fibrosis in systemic sclerosis. Genome Med. 2017;9(1):27.

2. Bossini-Castillo L, Simeon CP, Beretta L, Vonk MC, Callejas-Rubio JL, Espinosa $\mathrm{G}$, et al. Confirmation of association of the macrophage migration inhibitory factor gene with systemic sclerosis in a large European population. Rheumatology (Oxford). 2011;50(11):1976-81. https://doi.org/10.1093/ rheumatology/ker259.

3. Lescoat A, Ballerie A, Augagneur Y, Morzadec C, Vernhet L, Fardel O, et al. Distinct properties of human M-CSF and GM-CSF monocyte-derived macrophages to simulate pathological lung conditions in vitro: application to systemic and inflammatory disorders with pulmonary involvement. Int J Mol Sci. 2018;19(3):894.

4. Becker H, Willeke P, Schotte H, Domschke W, Gaubitz M. Macrophage migration inhibitory factor may contribute to vasculopathy in systemic sclerosis. Clin Rheumatol. 2008;27(10):1307-11. https://doi.org/10.1007/s1 0067-008-0960-7.

5. Lescoat A, Lecureur V, Roussel M, Sunnaram BL, Ballerie A, Coiffier G, et al. CD16-positive circulating monocytes and fibrotic manifestations of systemic sclerosis. Clin Rheumatol. 2017;36(7):1649-54. https://doi.org/10.1007/s1 0067-017-3597-6.

6. Ziegler-Heitbrock HW, Ströbel M, Kieper D, Fingerle G, Schlunck T, Petersmann I, et al. Differential expression of cytokines in human blood monocyte subpopulations. Blood. 1992;79(2):503-11. https://doi.org/10.11 82/blood.V79.2.503.503

7. Cros J, Cagnard N, Woollard K, Patey N, Zhang SY, Senechal B, et al. Human CD14dim monocytes patrol and sense nucleic acids and viruses via TLR7 and TLR8 receptors. Immunity. 2010;33(3):375-86. https://doi.org/10.1016/j. immuni.2010.08.012.

8. Auffray C, Sieweke MH, Geissmann F. Blood monocytes: development, heterogeneity, and relationship with dendritic cells. Annu Rev Immunol. 2009;27(1):669-92. https://doi.org/10.1146/annurev.immunol.021908.132557.

9. Wong KL, Yeap WH, Tai JJ, Ong SM, Dang TM, Wong SC. The three human monocyte subsets: implications for health and disease. Immunol Res. 2012; 53(1-3):41-57. https://doi.org/10.1007/s12026-012-8297-3.

10. Passlick B, Flieger D, Ziegler-Heitbrock HW. Identification and characterization of a novel monocyte subpopulation in human peripheral blood. Blood. 1989;74(7):2527-34. https://doi.org/10.1182/ blood.V74.7.2527.2527.

11. González-Domínguez É, Domínguez-Soto Á, Nieto C, Flores-Sevilla JL, Pacheco-Blanco M, Campos-Peña V, et al. Atypical activin a and IL-10 production impairs human CD16+ monocyte differentiation into antiinflammatory macrophages. J Immunol. 2016;196(3):1327-37. https://doi. org/10.4049/jimmunol.1501177.

12. van Furth R. Origin and turnover of monocytes and macrophages. Curr Top Pathol. 1989;79:125-50. https://doi.org/10.1007/978-3-642-73855-5_6.

13. Christmann RB, Hayes E, Pendergrass S, Padilla C, Farina G, Affandi AJ, et al. Interferon and alternative activation of monocyte/macrophages in systemic sclerosis-associated pulmonary arterial hypertension. Arthritis Rheum. 2011; 63(6):1718-28. https://doi.org/10.1002/art.30318.

14. Gundra UM, Girgis NM, Ruckerl D, Jenkins S, Ward LN, Kurtz ZD, et al. Alternatively activated macrophages derived from monocytes and tissue macrophages are phenotypically and functionally distinct. Blood. 2014; 123(20):e110-22. https://doi.org/10.1182/blood-2013-08-520619.

15. York MR, Nagai T, Mangini AJ, Lemaire R, van Seventer JM, Lafyatis R. A macrophage marker, Siglec-1, is increased on circulating monocytes in patients with systemic sclerosis and induced by type I interferons and tolllike receptor agonists. Arthritis Rheum. 2007;56(3):1010-20. https://doi.org/1 0.1002 /art.22382.

16. van den Hoogen F, Khanna D, Fransen J, Johnson SR, Baron M, Tyndall A, et al. 2013 classification criteria for systemic sclerosis: an American College of Rheumatology/European league against rheumatism collaborative initiative. Arthritis Rheum. 2013;65(11):2737-47. https://doi. org/10.1002/art.38098.

17. Johnson SR, Feldman BM, Hawker GA. Classification criteria for systemic sclerosis subsets. J Rheumatol. 2007;34(9):1855-63.

18. LeRoy EC, Medsger TA. Criteria for the classification of early systemic sclerosis. J Rheumatol. 2001;28(7):1573-6.

19. Clements $P$, Lachenbruch $P$, Siebold J, White B, Weiner S, Martin R, et al Inter and intraobserver variability of total skin thickness score (modified Rodnan TSS) in systemic sclerosis. J Rheumatol. 1995;22(7):1281-5.

20. Wong KL, Tai JJ, Wong WC, Han H, Sem X, Yeap WH, et al. Gene expression profiling reveals the defining features of the classical, intermediate, and nonclassical human monocyte subsets. Blood. 2011;118(5):e16-31. https:// doi.org/10.1182/blood-2010-12-326355.

21. Roederer M. Compensation in flow cytometry. Curr Protoc Cytom. 2002; Chapter 1:Unit 1.14.

22. Higashi-Kuwata N, Jinnin M, Makino T, Fukushima S, Inoue Y, Muchemwa FC, et al. Characterization of monocyte/macrophage subsets in the skin and peripheral blood derived from patients with systemic sclerosis. Arthritis Res Ther. 2010;12(4):R128. https://doi.org/10.1186/ar3066.

23. Rossol M, Kraus S, Pierer M, Baerwald C, Wagner U. The CD14(bright) CD16+ monocyte subset is expanded in rheumatoid arthritis and promotes expansion of the Th17 cell population. Arthritis Rheum. 2012;64(3):671-7. https://doi.org/10.1002/art.33418.

24. Cavaliere FM, Prezzo A, Bilotta C, lacobini M, Quinti I. The lack of BTK does not impair monocytes and polymorphonuclear cells functions in X-linked agammaglobulinemia under treatment with intravenous immunoglobulin replacement. PLoS One. 2017;12(4):e0175961. https://doi.org/10.1371/journal. pone.0175961.

25. Burbano C, Vasquez G, Rojas M. Modulatory effects of CD14+CD16++ monocytes on CD14++CD16- monocytes: a possible explanation of monocyte alterations in systemic lupus erythematosus. Arthritis Rheum. 2014;66(12):3371-81. https://doi.org/10.1002/art.38860.

26. Mahoney JM, Taroni J, Martyanov V, Wood TA, Greene CS, Pioli PA, et al. Systems level analysis of systemic sclerosis shows a network of immune and profibrotic pathways connected with genetic polymorphisms. PLoS Comput Biol. 2015;11(1):e1004005. https://doi.org/10.1371/journal.pcbi.1004005.

27. Lambert C, Preijers FWMB, Yanikkaya Demirel G, Sack U. Monocytes and macrophages in flow: an ESCCA initiative on advanced analyses of monocyte lineage using flow cytometry. Cytometry B Clin Cytom. 2017; 92(3):180-8.

28. Yona S, Kim KW, Wolf Y, Mildner A, Varol D, Breker M, et al. Fate mapping reveals origins and dynamics of monocytes and tissue macrophages under homeostasis. Immunity. 2013;38(1):79-91. https://doi.org/10.1016/j.immuni.2 012.12.001.

29. Davies LC, Rosas M, Jenkins SJ, Liao CT, Scurr MJ, Brombacher F, et al. Distinct bone marrow-derived and tissue-resident macrophage lineages proliferate at key stages during inflammation. Nat Commun. 2013;4(1):1886. https://doi.org/10.1038/ncomms2877.

30. Hashimoto D, Chow A, Noizat C, Teo P, Beasley MB, Leboeuf M, et al. Tissueresident macrophages self-maintain locally throughout adult life with minimal contribution from circulating monocytes. Immunity. 2013;38(4): 792-804. https://doi.org/10.1016/j.immuni.2013.04.004.

31. Jenkins SJ, Ruckerl D, Cook PC, Jones LH, Finkelman FD, van Rooijen N, et al. Local macrophage proliferation, rather than recruitment from the blood, is a signature of TH2 inflammation. Science. 2011;332(6035):1284-8. https://doi. org/10.1126/science.1204351.

32. Trombetta AC, Soldano S, Contini P, Tomatis V, Ruaro B, Paolino S, et al. A circulating cell population showing both $\mathrm{M} 1$ and $\mathrm{M} 2$ monocyte/ macrophage surface markers characterizes systemic sclerosis patients with lung involvement. Respir Res. 2018;19(1):186. https://doi.org/10.1186/s12931018-0891-z. 
33. Matzinger P, Kamala T. Tissue-based class control: the other side of tolerance. Nat Rev Immunol. 2011;11(3):221-30.

34. Soldano S, Trombetta AC, Contini P, Tomatis V, Ruaro B, Brizzolara R, et al. Increase in circulating cells coexpressing M1 and M2 macrophage surface markers in patients with systemic sclerosis. Ann Rheum Dis. 2018;77(12): 1842-5. https://doi.org/10.1136/annrheumdis-2018-213648.

35. Bogie JF, Boelen E, Louagie E, Delputte P, Elewaut D, van Horssen J, et al. CD169 is a marker for highly pathogenic phagocytes in multiple sclerosis. Mult Scler. 2018;24(3):290-300. https://doi.org/10.1177/1352458517698759.

36. Xiong YS, Cheng Y, Lin QS, Wu AL, Yu J, Li C, et al. Increased expression of Siglec-1 on peripheral blood monocytes and its role in mononuclear cell reactivity to autoantigen in rheumatoid arthritis. Rheumatology (Oxford). 2014;53(2):250-9. https://doi.org/10.1093/rheumatology/ket342.

37. Ikezumi Y, Suzuki T, Hayafuji S, Okubo S, Nikolic-Paterson DJ, Kawachi H, et al. The sialoadhesin (CD169) expressing a macrophage subset in human proliferative glomerulonephritis. Nephrol Dial Transplant. 2005;20(12):270413. https://doi.org/10.1093/ndt/gfi105.

38. Oetke C, Vinson MC, Jones C, Crocker PR. Sialoadhesin-deficient mice exhibit subtle changes in B- and T-cell populations and reduced immunoglobulin M levels. Mol Cell Biol. 2006;26(4):1549-57. https://doi. org/10.1128/MCB.26.4.1549-1557.2006.

39. Stifano G, Christmann RB. Macrophage involvement in systemic sclerosis: do we need more evidence? Curr Rheumatol Rep. 2016;18(1):2. https://doi. org/10.1007/s1 1926-015-0554-8.

\section{Publisher's Note}

Springer Nature remains neutral with regard to jurisdictional claims in published maps and institutional affiliations.

Ready to submit your research? Choose BMC and benefit from:

- fast, convenient online submission

- thorough peer review by experienced researchers in your field

- rapid publication on acceptance

- support for research data, including large and complex data types

- gold Open Access which fosters wider collaboration and increased citations

- maximum visibility for your research: over $100 \mathrm{M}$ website views per year

At $\mathrm{BMC}$, research is always in progress.

Learn more biomedcentral.com/submissions 